\title{
Tuning of Adaptive Weight Depth Map Generation Algorithms
}

\section{Exploratory Data Analysis and Design of Computer Experiments (DOCE)}

\author{
Diego Acosta - John Congote - Iñigo Barandiaran • Oscar Ruiz • \\ Alejandro Hoyos • Manuel Graña
}

Received: 01/11/2011 / Accepted: XX/XX/XXXX

Abstract In depth map generation algorithms, parameters settings to yield an accurate disparity map estimation are usually chosen empirically or based on unplanned experiments. Algorithms' performance is measured based on the distance of the algorithm results vs. the Ground Truth by Middlebury's standards. This work shows a systematic statistical approach including exploratory data analyses on over 14000 images and designs of experiments using 31 depth maps to measure the relative influence of the parameters and to fine-tune them based on the number of bad pixels. The implemented methodology improves the performance of adaptive weight based dense depth map algorithms. As a result, the algorithm improves from $16.78 \%$ to $14.48 \%$ bad pixels using a classical exploratory data analysis of over 14000 existing images, while using designs of computer experiments with 31 runs yielded an even better performance by lowering bad pixels from $16.78 \%$ to $13 \%$.

Keywords Stereo Image Processing; Parameter Estimation; Depth Map; Statistical Design of Computer Experiments

\section{Introduction}

Depth map calculation deals with the estimation of multiple object depths on a scene. It is useful for applications like vehicle navigation, automatic surveillance,

DDP Research Group, EAFIT University, Medellin, Colombia E-mail: dacostam@eafit.edu.co, · Vicomtech Research Center, Donostia-San Sebastián, Spain E-mail: ibarandiaran@vicomtech.org, · CAD CAM CAE Laboratory, EAFIT University, Medellin, Colombia E-mail: \{jcongote, ahoyossi, oruiz\}@eafit.edu.co · Dpto. CCIA, UPV-EHU E-mail: ccpgrrom@gmail.com aerial cartography, passive 3D scanning, automatic industrial inspection, or 3D videoconferencing [1]. These maps are constructed by generating, at each pixel, an estimation of the distance from the camera to the object surface (depth).

Disparity is commonly used to describe inverse depth in computer vision, and to measure the perceived spatial shift of a feature observed from close camera viewpoints. Stereo correspondence techniques often calculate a disparity function $d(x, y)$ relating target and reference images, so that the $(x, y)$ coordinates of the disparity space match the pixel coordinates of the reference image. Stereo methods commonly use a pair of images taken with a known camera geometry to generate a dense disparity map with estimates at each pixel. This dense output is useful for applications requiring depth values even in difficult regions like occlusions and textureless areas. The ambiguity of matching pixels in heavy textured or textureless zones tends to require complex and expensive global image processing or statistical correlations using color and proximity measures in local support windows.

Most implementations of vision algorithms make assumptions about the visual appearance of objects in the scene to ease the matching problem. The steps generally taken to compute the depth maps may include: (i) matching cost computation, (ii) cost or support aggregation, (iii) disparity computation or optimization, and (iv) disparity refinement.

This article is based on work done in 1] where the principles of stereo correspondence techniques and a quantitative evaluator are discussed. The literature review is presented in section 2 , followed by section 3 describing the algorithm, filters, statistical analyses and experimental set-up. Results and discussions are cov- 
ered in section 4, and the article is concluded with section 5

\section{Literature Review}

Depth-map generation algorithms and filters use several user-specified parameters to generate a depth map from an image pair. The settings of these algorithms are heavily influenced by the evaluated data sets 2 . Published works usually report the settings used for their specific case studies without describing the procedure followed to fine-tune them $3,4,5$, and some explicitly state the empirical nature of these values [6. The variation of the output as a function of several settings on selected parameters is briefly discussed while not taking into account the effect of modifying them all simultaneously [3,2,7]. Multiple stereo methods are compared choosing values based on experiments, but only some algorithm parameters are changed not explaining the rationale for setting them [1].

\subsection{Literature Review Conclusions}

Commonly used approaches in determining the settings of depth map algorithm parameters show all or some of the following shortcomings: (i) undocumented procedures for parameter setting, (ii) lack of planning when testing for the best settings, and (iii) failure to consider interactions of changing all the parameters simultaneously.

As a response to these disadvantages, this article presents a methodology to fine-tune user-specified parameters on a depth map algorithm using a set of images from the adaptive weight implementation in [4. Multiple settings are used and evaluated on all parameters to measure the contribution of each parameter to the output variance. A quantitative accuracy evaluation allows using main effects plots and analyses of variance on multi-variate linear regression models to select the best combination of settings for each data set. The initial results are improved by setting new estimated values of user-specified parameters, allowing the algorithm to give much more accurate results on a rectified image pair.

Since it is not always feaisible to have a large set of images available, a fractional factorial design of computer experiment (DOCE) with only eight runs is used to find out which parameters have a major influence on the images tested. To optimize the parameters and to have the lowest percentage of bad pixels a central composite DOCE with 23 runs is used with the most influential parameters found in the fractional factorial design. To the best of our knowledge the systematic and efficient application of DOCE in the field of depth maps generation has not been done yet.

\section{Methodology}

\subsection{Image Processing}

In adaptive weight algorithms [3,4], a window is moved over each pixel on every image row, calculating a measurement based on the geometric proximity and color similarity of each pixel in the moving window to the pixel on its center. Pixels are matched on each row based on their support measurement with larger weights coming from similar pixel colors and closer pixels. The horizontal shift, or disparity, is recorded as the depth value, with higher values reflecting greater shifts and closer proximity to the camera.

The strength of grouping by color $\left(f_{s}\left(c_{p}, c_{q}\right)\right)$ for pixels $p$ and $q$ is defined as the Euclidean distance between colors $\left(\Delta c_{p q}\right)$ by Equation (1). Similarly, grouping strength by distance $\left(f_{p}\left(g_{p}, g_{q}\right)\right)$ is defined as the Euclidean distance between pixel image coordinates $\left(\Delta g_{p q}\right)$ as per Equation (2). $\gamma_{c}$ and $\gamma_{p}$ are adjustable settings used to scale the measured color delta, represented as $a w_{-} c o l$ in the study, and window size represented as aw_win respectively.

$$
\begin{aligned}
& f_{s}\left(c_{p}, c_{q}\right)=\exp \left(-\frac{\Delta c_{p q}}{\gamma_{c}}\right) \\
& f_{p}\left(g_{p}, g_{q}\right)=\exp \left(-\frac{\Delta g_{p q}}{\gamma_{p}}\right)
\end{aligned}
$$

The matching cost between pixels shown in Equation (3) is measured by aggregating raw matching costs, using the support weights defined by Equations (1) and (2), in support windows based on both the reference and target images.

$$
=\frac{E\left(p, \bar{p}_{d}\right)}{\sum_{q \in N_{p}, \bar{q}_{d} \in N_{\bar{p}_{d}}} w(p, q) w\left(\bar{p}_{d}, \bar{q}_{d}\right) \sum_{c \in\{r, g, b\}}\left|I_{c}(q)-I_{c}\left(\bar{q}_{d}\right)\right|}
$$

where $w(p, q)=f_{s}\left(c_{p}, c_{q}\right) \cdot f_{p}\left(g_{p}, g_{q}\right), \bar{p}_{d}$ and $\bar{q}_{d}$ are the target image pixels at disparity $d$ corresponding to pixels $p$ and $q$ in the reference image, $I_{c}$ is the intensity on channels red $(r)$, green $(g)$, and blue $(b)$, and $N_{p}$ is the window centered at $p$ and containing all $q$ pixels. The size of this movable window $N$ is a derived parameter of (aw_win). Increasing the window size reduces the chance of bad matches at the expense of missing relevant scene features. 
Table 1 Input and Output Variables of Depth Maps Generation Algorithms

INPUT VARIABLES

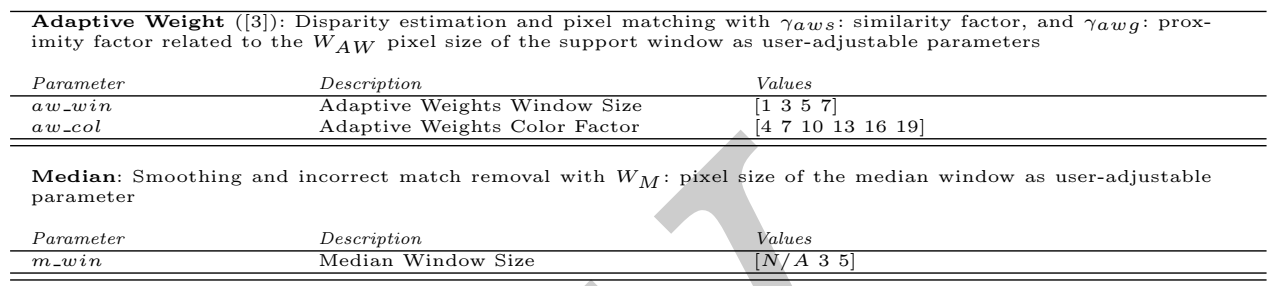

\begin{tabular}{l}
\hline \hline $\begin{array}{l}\text { Cross-check }([8]): \text { Validation of measurement per pixel with } \Delta_{d} \text { : allowed disparity difference as adjustable pa- } \\
\text { rameter } \\
\text { Parameter }\end{array}$ Description \\
\hline cc_disp $\quad$ Cross-Check Disparity Delta
\end{tabular}
Bilateral ([9]): Intensity and proximity weighted smoothing with edge preservation with $\gamma_{b s}$ : similarity factor,
and $\gamma_{b g}$ : proximity factor related to the $W_{B}$ pixel size of the bilateral window as user-adjustable parameters

\begin{tabular}{llllll} 
Parameter & Description & Values & & \\
\hline$c b \_w i n$ & Cross-Bilateral Window Size & {$\left[\begin{array}{lllllll}N / A & 1 & 3 & 5 & 7\end{array}\right]$} & & \\
$c b_{-}$col & Cross-Bilateral Color Factor & {$\left[\begin{array}{llllllll}N / A & 4 & 7 & 10 & 13 & 16 & 19\end{array}\right]$} \\
\hline
\end{tabular}

OUTPUT VARIABLES

\begin{tabular}{ll}
\hline rms_error_all & Root Mean Square (RMS) disparity error (all pixels) \\
rms_error_nonocc & RMS disparity error (non-occluded pixels only)
\end{tabular}

rms_error_nonocc $\quad$ RMS disparity error (non-occluded pixels only)

$\begin{array}{ll}\text { rms_error_occ } & \text { RMS disparity error (occluded pixels only) } \\ \text { rms_error_textured } & \text { RMS disparity error (textured pixels only) }\end{array}$

rms_error_textureless $\quad$ RMS disparity error (textureless pixels only) rms_error_discont RMS disparity error (near depth discontinuities)

\begin{tabular}{ll}
\hline bad_pixels_all & Fraction of bad points (all pixels) \\
bad_pixels_nonocc & Fraction of bad points (non-occluded pixels only)
\end{tabular}

$\begin{array}{ll}\text { bad_pixels_nonocc } & \text { Fraction of bad points (non-occluded pixels on } \\ \text { bad-pixels_occ } & \text { Fraction of bad points (occluded pixels only) }\end{array}$

bad_pixels_textured $\quad$ Fraction of bad points (textured pixels only)

bad-pixels-textureless $\quad$ Fraction of bad points (textureless pixels only)

\subsection{Post-Processing Filters}

Algorithms based on correlations depend heavily on finding similar textures at corresponding points in both reference and target images. Bad matches happen more frequently in textureless regions, occluded zones, and areas with high variation in disparity, such as discontinuities. The winner takes all approach enforces uniqueness of matches only for the reference image in such a way that points on the target image may be matched more than once, creating the need to check the disparity estimates and fill any gaps with information from neighboring pixels using post-processing filters like the ones shown on Table 1 .

Median Filter $(m)$. This filter is widely used in digital image processing to smooth signals and to remove incorrect matches and holes by assigning neighboring disparities at the expense of edge preservation. The median filter provides a mechanism for reducing image noise, while preserving edges more effectively than a linear smoothing filter. It sorts the intensities of all $q$ pixels on a window of size $M$ and selects the median value as the new intensity of the $p$ central pixel. The size $M$ of the window is another of the user-specified parameters.

Cross-check Filter(cc). The correlation is performed twice by reversing the roles of the two images (reference and target) and considering valid only those matches having similar depth measures at corresponding points in both steps. The validity test is prone to fail in occluded areas where disparity estimates will be rejected. The allowed difference in disparities between reference and target images is one more adjustable parameter.

Bilateral Filter $(c b)$. Is a non-iterative method of smoothing images while retaining edge detail. The intensity value at each pixel in an image is replaced by a weighted average of intensity values from nearby pixels. The weighting for each pixel $q$ is determined by the spatial distance from the center pixel $p$, as well as its relative difference in intensity, defined by Equation (4).

$O_{p}=\frac{\sum_{q \in W} f_{s}(q-p) g_{i}\left(I_{q}-I_{p}\right) I_{q}}{\sum_{q \in W} f_{s}(q-p) g_{i}\left(I_{q}-I_{p}\right)}$

$O_{p}$ is the output image, $I$ the input image, $W$ the weighting window, $f_{s}$ the spatial weighting function, and $g_{i}$ the intensity weighting function. The size of the window $W$ is yet another parameter specified by the user.

\subsection{Experimental Set-up}

The depth maps of our experiments are calculated with an implementation developed for real time videoconferencing in [4]. Using well-known rectified image sets: 
Cones from [1], Teddy and Venus from [10, and Tsukuba head and lamp from the University of Tsukuba. Other commonly used sets are also freely available [11,12. Our dataset consists of 14688 depth maps, 3672 for each data set, like the ones shown on Figure 1.

Many recent stereo correspondence performance studies use the Middlebury Stereomatcher for their quantitative comparisons [2,7,13. The evaluator code, sample scripts, and image data sets are available from the Middlebury stereo vision site [16], providing a flexible and standard platform for easy evaluation.

The online Middlebury Stereo Evaluation Table gives a visual indication of how well the methods perform with the proportion of bad pixels (bad_pixels) metric defined as the average of the proportion of bad pixels in the whole image (bad_pixels_all), the proportion of bad pixels in non-occluded regions (bad_pixels_nonocc) and the proportion of bad pixels in areas near depth discontinuities (bad_pixels_discont) in all data sets. A bad pixel represents a pixel where the estimated disparity is wrong with respect to a ground thruth disparity value.

\subsection{Statistical Analyses}

The user-specified input parameters and output accuracy data are statistically analyzed to correlate them (see Table 1). Box plots give insights on the influence of settings on a given response variable. A multi-variate regression model shown in Equation (5) relates the output variable as a function of all the parameters to find the equation coeffcients, correlation of determination, and allows the analysis of variance to measure the influence of each parameter on the output variance, where $\hat{y}$ hat is the predicted response variable. $x_{i}$ are the input factors, $\beta_{0}$ and $\beta_{i}$ are the coefficients fit by multi-variable linear regression. Residuals are analyzed to validate the assumptions of the regression model (i.e. constant variance of residuals, null mean of residuals). When those assumptions are not fulfilled, the model is modified [17. The parameters are normalized to fit the range $(-1,1)$ at their values shown on Table 1.

$\hat{y}=\beta_{0}+\sum_{i=1}^{n} \beta_{i} x_{i}+\epsilon$

Having a large data set (in this case 14688 images) to perform statistical analyses is not always feasible. DOCE is applied here to obtain an equivalently good model for the depth map, by having a much smaller number of runs. A $2^{6-3}$ fractional factorial DOCE with just eight runs allows to establish which ones of the parameters $a w \_w i n, a w \_c o l o, m_{-} \_$win, $c c_{-} d i s p, c b \_w i n$, and $c b \_c o l$ are the most influential on the bad_pixels output by using a Daniel plot 14. The parameters whose distribution cannot be considered as normal standard are statistically relevant in the fractional DOCE. Therefore, they are used to optimize the depth map generation algorithm.

A surface response central composite DOCE with 23 runs was performed afterward with aw_win, aw_colo, m_win, and cb_win as studied factors while keeping constant the remaining parameters (i.e., $c c \_d i s p=2$ y $\left.c b \_c o l=13\right)$ to yield a mathematical model of the form:

$\hat{y}=\beta_{0}+\sum_{i}^{k} \beta_{i} x_{i}+\sum_{i i}^{k} \beta_{i i} x_{i}^{2}+\sum_{i<j} \beta_{i j} x_{i} x_{j}$

where, as in equation 5, $\hat{y}$ is the predicted variable. The $x_{i}$ are the parameters. $\beta_{0}, \beta_{i}, \beta_{i i}$ and $\beta_{i j}$ are constants adjusted by minimum least squares regression. Data from DOCE was analysed with the software for statistical computing $\mathrm{R}$ with Bayes Screening and Model Discrimination -BsMD- and Response Surface Method -RSM- add-on packages [15.

\section{Results and Discussion}

\subsection{Selection of Input Variables for Mathematical}

Model.

Response variables for depth map generation algorithms are shown with their meaning on Table 1. Pearson multiple correlation coefficients for the response variables shown on Table 2 shows that bad_pixels_all is strongly correlated to the remaining response variables. This means that all response variables follow a similar trend as bad_pixels_all and that modeling bad_pixels_all is sufficient to reach statistically sound results for depth map generation algorithms optimization.

On the other hand, low Pearson coefficients for the input variables indicate that those variables are independent, that there is no co-linearity among them and that each independent variable must be included in the exploratory analysis.

\subsection{Exploratory Data Analysis}

Box plots analyses of bad_pixels presented on Figure2 shows lower output values from using filters, relaxed cross-check disparity delta values, large adaptive weight window sizes, and large adaptive weight color factor values. The median window size, bilateral window size, and bilateral window color values do not show a significant influence on the output at the studied levels. 


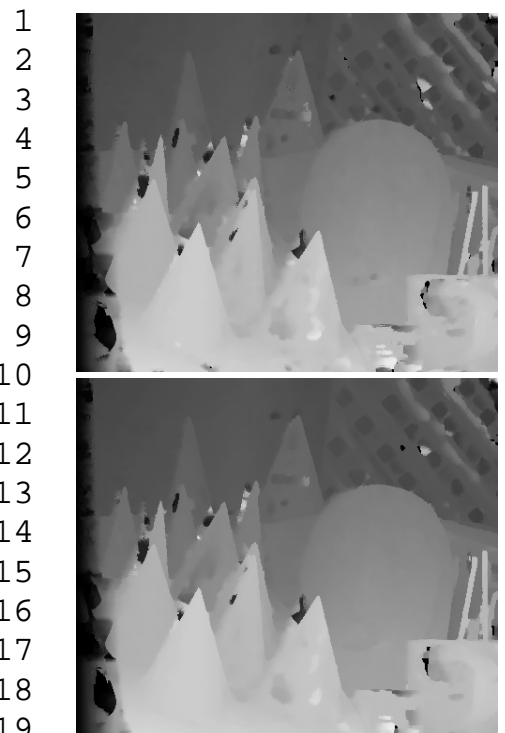

(a)

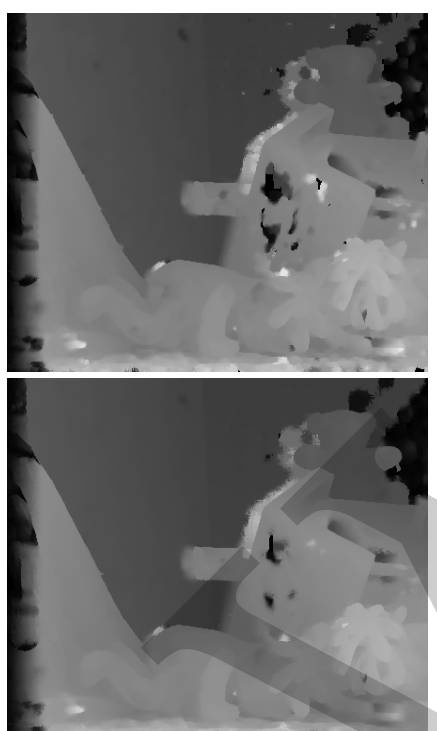

(b)

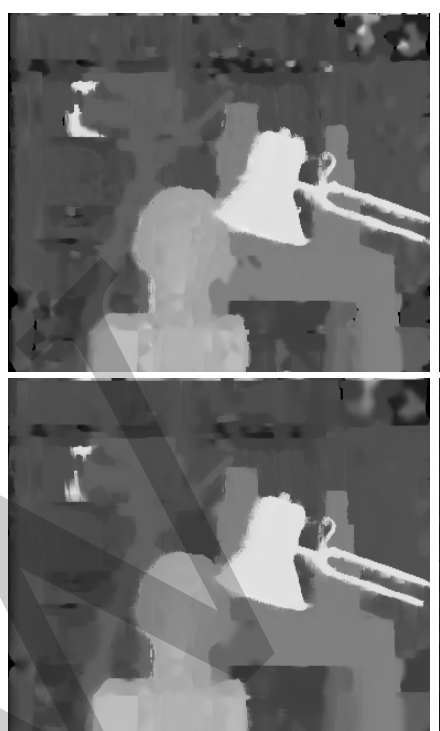

(c)

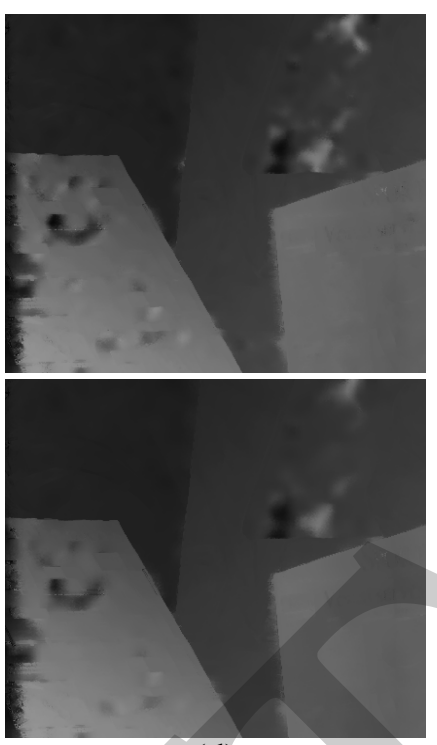

(d)

Fig. 1 Depth Map Comparison. Top: best initial, bottom: new settings. (a) Cones, (b) Teddy, (c) Tsukuba, and (d) Venus data set.

Table 2 Pearson correlation coefficient for the evaluator outputs over all data sets

\begin{tabular}{|c|c|c|c|c|c|c|c|c|c|c|c|c|c|}
\hline & (1) & (2) & (3) & $(4)$ & (5) & (6) & (7) & $(8)$ & (9) & (10) & (11) & $(12)$ & (13) \\
\hline (1)bad_pixels & 1.00 & 0.81 & $\overline{0.82}$ & 0.59 & 0.83 & 0.77 & 0.84 & 1.00 & 1.00 & 0.86 & 1.00 & 0.95 & 0.99 \\
\hline (2)rms_error_all & 0.81 & 1.00 & 1.00 & 0.69 & 1.00 & 0.98 & 0.99 & 0.82 & 0.82 & 0.64 & 0.85 & 0.70 & 0.79 \\
\hline (3)rms_error_nonocc & 0.82 & 1.00 & 1.00 & 0.71 & 1.00 & 0.98 & 0.99 & 0.83 & 0.82 & 0.67 & 0.85 & 0.71 & 0.80 \\
\hline (4)rms_error_occ & 0.59 & 0.69 & 0.71 & 1.00 & 0.70 & 0.77 & 0.74 & 0.62 & 0.61 & 0.68 & 0.61 & 0.63 & 0.53 \\
\hline (5)rms_error_textured & 0.83 & 1.00 & 1.00 & 0.70 & 1.00 & 0.98 & 0.99 & 0.83 & 0.83 & 0.67 & 0.86 & 0.72 & 0.81 \\
\hline (6)rms_error_textureless & 0.77 & 0.98 & 0.98 & 0.77 & 0.98 & 1.00 & 0.98 & 0.78 & 0.78 & 0.64 & 0.80 & 0.68 & 0.73 \\
\hline (7)rms_error_discont & 0.84 & 0.99 & 0.99 & 0.74 & 0.99 & 0.98 & 1.00 & 0.85 & 0.84 & 0.67 & 0.87 & 0.73 & 0.82 \\
\hline (8)bad_pixels_all & 1.00 & 0.82 & 0.83 & 0.62 & 0.83 & 0.78 & 0.85 & 1.00 & 1.00 & 0.85 & 1.00 & 0.96 & 0.98 \\
\hline (9) bad_pixels_nonocc & 1.00 & 0.82 & 0.82 & 0.61 & 0.83 & 0.78 & 0.84 & 1.00 & 1.00 & 0.85 & 1.00 & 0.96 & 0.98 \\
\hline (10)bad_pixels_occ & 0.86 & 0.64 & 0.67 & 0.68 & 0.67 & 0.64 & 0.67 & 0.85 & 0.85 & 1.00 & 0.83 & 0.87 & 0.86 \\
\hline (11)bad_pixels_textured & 1.00 & 0.85 & 0.85 & 0.61 & 0.86 & 0.80 & 0.87 & 1.00 & 1.00 & 0.83 & 1.00 & 0.93 & 0.99 \\
\hline (12) bad_pixels_textureless & 0.95 & 0.70 & 0.71 & 0.63 & 0.72 & 0.68 & 0.73 & 0.96 & 0.96 & 0.87 & 0.93 & 1.00 & 0.93 \\
\hline (13)bad_pixels_discont & 0.99 & 0.79 & 0.80 & 0.53 & 0.81 & 0.73 & 0.82 & 0.98 & 0.98 & 0.86 & 0.99 & 0.93 & 1.00 \\
\hline
\end{tabular}

The influence of the parameters is also shown on the slopes of the main effects plots of Figure 3 and confirms the behavior found with the analysis of variance (ANOVA) of the multi-variate linear regression model. The optimal settings from this analysis (i.e., $a w_{\_} w i n=9, a w_{-} c o l=22, m_{-}$win $=5, c c_{-} d i s p=1$, $c b \_w i n=3$ and $\left.c b \_c o l=4\right)$ to minimize bad_pixels yields a result of $14.48 \%$.

\subsection{Multi-variate Linear Regression Model.}

The analysis of variance on a multi-variate linear regression (MVLR) over all data sets using the most parsimonious model quantifies the parameters with the most influence as shown on Table 3 . The most significant input variable is $c c_{-} d i s p$, since it accounts for a [33\%-50\%] of the variance in every case.

Interactions and higher order terms are included on the multi-variate linear regression models to improve the goodness of fit. Reducing the number of input images per dataset from 3456 to 1526 by excluding the worst performing cases $\left(c c_{-} d i s p=0, a w_{-} c o l=4\right.$ and
Table 3 Linear model ANOVA with the contribution to the sum of squared errors (SSE) of bad_pixels.

\begin{tabular}{lrrrr} 
Data set & cc_disp & aw_win & aw_col & cb_win \\
\hline Cones & $34.35 \%$ & $14.46 \%$ & $17.47 \%$ & - \\
Teddy & $41.25 \%$ & $13.75 \%$ & $8.10 \%$ & - \\
Tsukuba & $50.25 \%$ & - & - & $7.16 \%$ \\
Venus & $47.35 \%$ & $9.42 \%$ & - & $5.62 \%$ \\
All & $47.01 \%$ & $8.11 \%$ & - & -
\end{tabular}

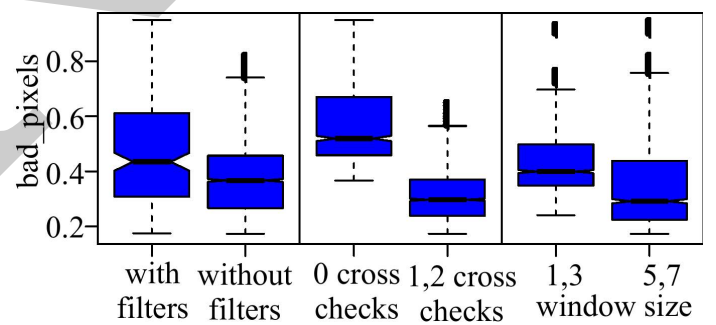

Fig. 2 Box Plots for Input Variable Analysis

$\left.a w_{-} c o l=7\right)$, using a cubic model with interactions yields a very good multiple correlation coefficient of $R^{2}=99.05 \%$. However, for the model selected the residuals distribution is not normal even after transforming the response variable and removing large residuals val- 
ues. Another constraint for the statistical analyses is that any outliers from the data set cant be excluded. Nonetheless, improved algorithm performance settings are found using the model to obtain lower bad_pixels values comparable to the ones obtained through the exploratory data analysis (14.66\% vs. $14.48 \%)$.

In summary, the most noticeable influence on the output variable comes from having a relaxed cross-check filter, accounting for nearly half the response variance in all the study data sets. Window size is the next most influential factor, followed by color factor, and finally window size on the bilateral filter. Increasing the window size on the main algorithm yields better overall results at the expense of longer running times and some foreground loss of sharpness, while the support weights on each pixel have the chance of becoming more distinct and potentially reduce disparity mismatches. Increasing the color factor on the main algorithm allows better results by reducing the color differences, and slightly compensating minor variations in intensity from different viewpoints.

A small median smoothing filter window size is faster than a larger one, while still having a similar accuracy. Low settings on both the window size and the color factor on the bilateral filter seem to work best for a good trade-off between performance and accuracy.

The optimal settings in the original data set are presented on Table 4 along with the proposed settings. Low settings comprise the depth maps with all their parameter settings at each of their minimum tested values yielding $67.62 \%$ bad_pixels. High settings relates to depth maps with all their parameter settings at each of their maximum tested values yielding $19.84 \%$ bad_pixels. Best initial are the most accurate depth maps from the study data set yielding $16.78 \%$ bad_pixels. Exploratory analysis corresponds to the settings determined using the exploratory data analysis based on box plots and main effects plots yielding $14.48 \%$ bad_pixels.

MVLR optimization is the optimization of the classical data analysis based on multi-variate linear regression model, nested models, and ANOVA yielding $14.66 \%$ bad_pixels.

The exploratory analysis estimation and the MVLR optimization tend to converge at similar lower bad_pixels values using the same image data set. The best initial and improved depth map outputs are shown in Figure 1. The best runs for fractional factorial and central composite DOCEs lower the value of the bad_pixels variable to $14.72 \%$ and $13.05 \%$, respectively. Notice that to achieve these results only 31 depth maps are needed (DOCE) as opposed to analyzing over 14000 depth maps (Exploratory Analysis).
4.4 Depth-map optimization by design of computer experiments (DOCE)

\section{$2^{6-3}$ Fractional Factorial Design of Experiment.}

The goal of this type of design of experiment is to screen the statistically most significant parameters. Details on how to set up the runs are discussed in [17. The design matrix describing all experimental runs can be set so that the high and low levels for each parameter are chosen by assigning them the maximum and minimum values allowed by the algorithm respectively. This was done for all of the parameters but for m_win (i.e., it was set at the levels 3 and 5), to avoid bias from the results and conclusions obtained from the exploratory and multivariate regression analysis. The results for this DOCE range from $14.72 \%$ and $72.17 \%$ bad pixels for all images which is quite promising because already with only eight runs a set of parameters values that is very close to the optimum obtained by exploratory analysis of $14.48 \%$ bad pixels and the multivariate linear regression analysis of $14.66 \%$ on the 14688 data points is delivered. The alias for the parameters and Daniel plot showing the most relevant parameters are shown on Figure 4

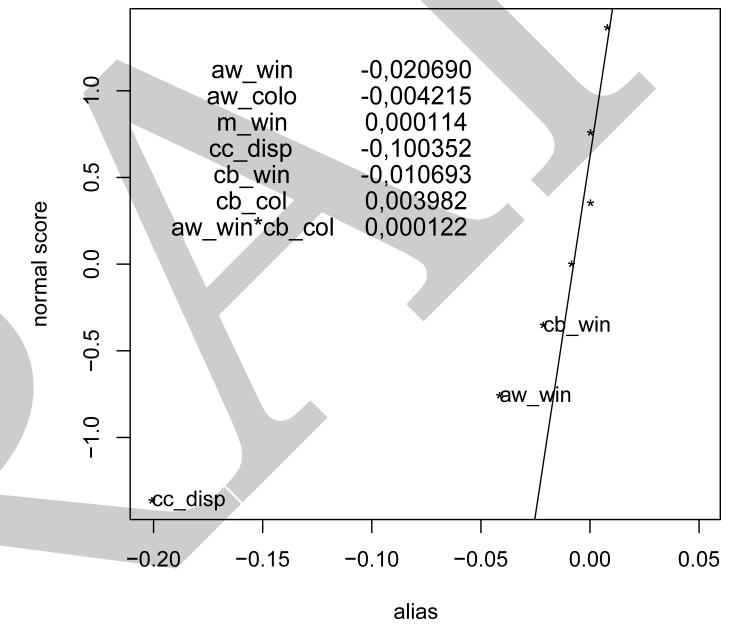

Fig. 4 Daniel Plot for determining the significance of input variables.

Daniel's plot indicate that the most influential parameters are $c c \_d i s p, a w \_w i n$ and $c b \_w i n$ which deviate the most from the normal distribution curve. These parameters and $m_{-}$win at levels 0,3 and 5 are used for the surface response methodology central composite design of experiment that follows.

Central Composite Design of Experiment.

To further optimize the depth maps generation algorithm a central composite design of experiment is used. As with the fractional factorial design of experi- 


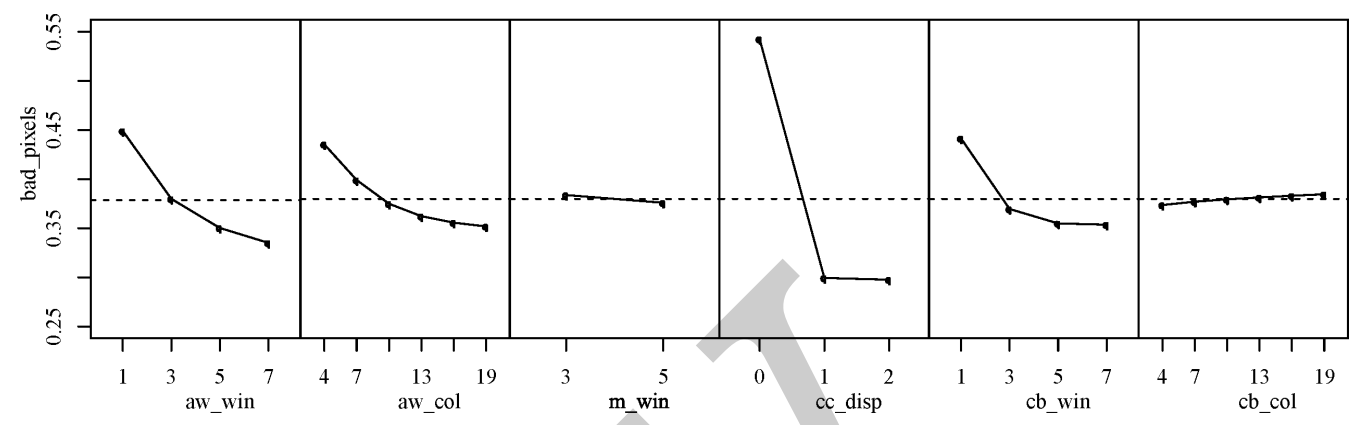

Fig. 3 Main Effects Plots of each factor level for all data sets. Steeper slopes relate to bigger influence on the variance of the bad_pixels output measurement.

Table 4 Model comparison. Average bad_pixels

\begin{tabular}{|c|c|c|c|c|c|c|}
\hline Run Type & bad_pixels & $a w \_w i n$ & $a w_{-} c o l$ & m_win & cc_disp & $c b \_w i \gamma$ \\
\hline Low Settings & $67.62 \%$ & 1 & 4 & 3 & 0 & \\
\hline High Settings & $19.84 \%$ & 7 & 19 & 5 & 2 & \\
\hline Best Initial & $16.78 \%$ & 7 & 19 & 5 & 1 & \\
\hline Exploratory analysis & $14.48 \%$ & 9 & 22 & 5 & 1 & \\
\hline MVLR optimization & $14.66 \%$ & 11 & 22 & 5 & 3 & \\
\hline Best Treatment for & & & & & & \\
\hline Fractional Factorial DOCE & $14.72 \%$ & 10 & 25 & 3 & 3 & \\
\hline Best Treatment for CCD DOCE & $13.05 \%$ & 7 & 14 & 3 & 4 & \\
\hline
\end{tabular}

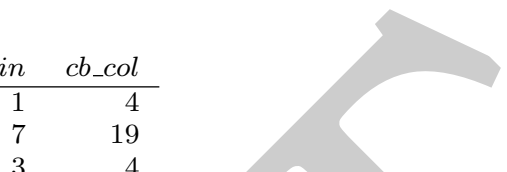

ment, the best run with $13.05 \%$ bad pixels is obtained amongst the 23 treatments which surpasses the results obtained thus far. The outputs from R [18] using the rsm package at the levels tested for each parameter are shown in Table 5 .

As it can be seen the second order model depicted before in equation 6 fits very well the data as indicated by the multiple correlation coefficient 0.9695 . The most significant variables include aw_win, aw_col, m_win, cb_win, aw_win ${ }^{2}, a w_{-} c o l^{2}$, and $m_{-} w_{i n}{ }^{2}$. Nonetheless, the complete model with all coefficients is used to draw the contour plots shown later. The rsm package also allows to detect stationary points. In this case the stationary point detected is a saddle point because one of the eigen-values is negative while the remaining ones are positive

Graphically the iso-lines for bad_pixels_all are seen in slices by looking at two parameters simultaneously for the analysis while keeping the remaining ones constant as shown on Figure 5. The graphs allow to see that the stationary point does indicate a local minimum when analyzing for aw_win and aw_col. With $m_{-}$win though, the graph indicates that a saddle is detected and that it is better to use values not in the $1: 5<m_{-}$win $<3: 5$ interval (which is physically imposible). For $c b_{-}$win the stationary point apparently corresponds to a minimum. The settings for the stationary point closer to what rsm's package detects are $a w \_s i z e=7, a w \_c o l=14, m_{-}$size $=3, c c \_d i s p=2$, $c b_{-}$size $=21$ and $c b_{-} c o l=13$ and this yields $26 \%$

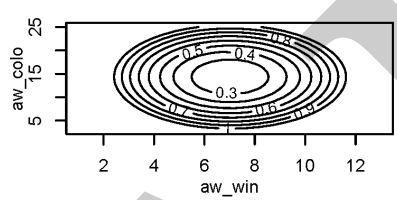

aw_win
Slice at $\mathrm{m} \_$win $=2.65, \mathrm{cb} \_$win $=7$

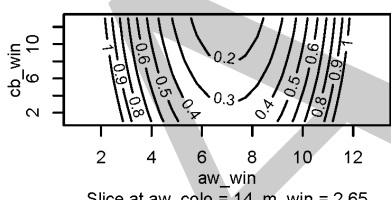

Slice at aw_colo $=14, \mathrm{~m}_{-}$win $=2.65$
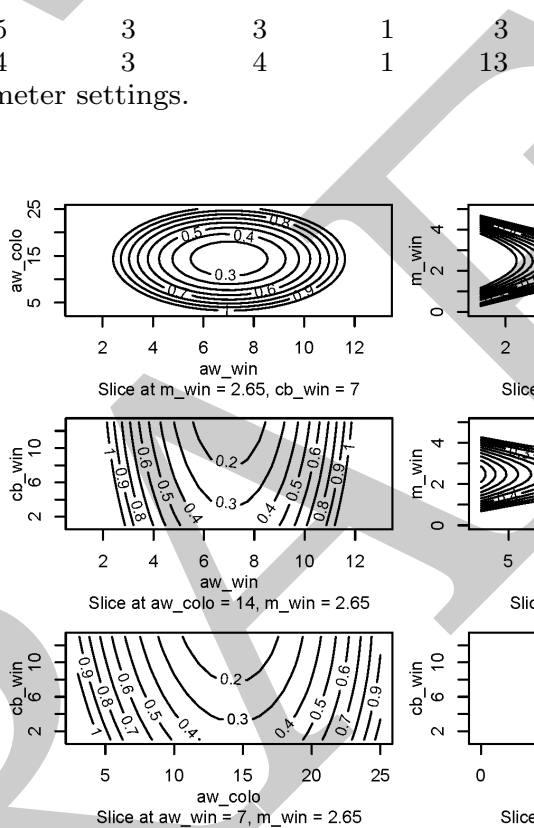

Fig. 5 Contour Plots for Central Composite DOCE.

bad_pixels_all leading to conclude that the best treatment for the rsm yielding $13.05 \%$ of bad_pixels_all is the local minimum optimum at the settings shown on Table 4

\section{Conclusions and Future Work}

Previously published material [19] showed how Exploratory Analysis, applied on over 14000 images, allowed the sub-optimal tuning of the parameters for Disparity Estimation algorithms, lowering the percentage of bad pixels from from $16.78 \%$ (manual tuning) to $14.48 \%$. 
Table 5 Summary of RSM Central Composite DOCE

\begin{tabular}{|c|c|c|c|c|c|}
\hline \multicolumn{2}{|l|}{ Parameters } & \multicolumn{2}{|c|}{ Levels } & & \\
\hline aw_win & 1 & 4 & 7 & 10 & \\
\hline aw_colo & 3 & 8.5 & 14 & 19.5 & 25 \\
\hline m_win & 0 & 3 & 5 & & \\
\hline$c b_{-} w i n$ & 1 & 4 & 7 & 10 & 13 \\
\hline $\mathrm{cb}_{\mathrm{C}} \mathrm{colo}$ & 13 & & & & \\
\hline$c c_{-}$disp & 2 & & & & \\
\hline \multicolumn{6}{|c|}{ Call: $\operatorname{rsm}\left(\right.$ formula $=$ bad_pixels_all $\sim \mathrm{SO}\left(a w_{-}\right.$win $\left.\left., a w_{-} c o l o, m_{-} w_{i n}, c b_{-} w i n\right)\right)$} \\
\hline Coefficients & Estimate & Std. Error & t-value & $p>|t|$ & Signif \\
\hline (Intercept) & 1.634 & $2.49 \times 10^{-1}$ & 6.561 & 0.00018 & \\
\hline$a w_{-}$win & $-5.25 \times 10^{-1}$ & $8.03 \times 10^{-2}$ & -6.538 & 0.00018 & \\
\hline aw_colo & $-1.9 \times 10^{-1}$ & $4.78 \times 10^{-2}$ & -3.971 & 0.00411 & \\
\hline m_win & 1.606 & $4.22 \times 10^{-1}$ & 3.802 & 0.00522 & \\
\hline$c b_{-} w i n$ & $-3.96 \times 10^{-2}$ & $8.03 \times 10^{-2}$ & -0.493 & 0.63495 & \\
\hline$a w_{-} w i n: a w_{-} c_{c o l o}$ & $4.15 \times 10^{-5}$ & $1.59 \times 10^{-4}$ & 0.26 & 0.80128 & \\
\hline aw_win:m_win & $-6.13 \times 10^{-5}$ & $7.01 \times 10^{-4}$ & -0.087 & 0.93243 & \\
\hline$a w_{\text {_win: }} c b_{-} w i n$ & $1.73 \times 10^{-4}$ & $2.92 \times 10^{-4}$ & 0.592 & 0.56990 & \\
\hline aw_colo: $m_{-} w i n$ & $3.01 \times 10^{-4}$ & $3.82 \times 10^{-4}$ & 0.788 & 0.45339 & \\
\hline$a w_{-} c_{c o l o:} c_{-} w_{\text {win }}$ & $5.33 \times 10^{-4}$ & $1.59 \times 10^{-4}$ & 3.347 & 0.01013 & * \\
\hline$m_{-}$win:cb_win & $5.56 \times 10^{-4}$ & $7.01 \times 10^{-4}$ & 0.793 & 0.45083 & \\
\hline$a w_{-} w_{i n}{ }^{2}$ & $3.73 \times 10^{-2}$ & $5.73 \times 10^{-3}$ & 6.508 & 0.00019 & \\
\hline$a w_{-}$colo`$_{2} 2$ & $6.44 \times 10^{-3}$ & $1.70 \times 10^{-3}$ & 3.78 & 0.00539 & \\
\hline m_win^2 & $-3.25 \times 10^{-1}$ & $8.45 \times 10^{-2}$ & -3.846 & 0.00490 & * \\
\hline$c b_{-} w i n^{\wedge} 2$ & $7.19 \times 10^{-4}$ & $5.73 \times 10^{-3}$ & 0.126 & 0.90314 & \\
\hline \multirow{4}{*}{\multicolumn{6}{|c|}{$\begin{array}{l}\text { Signifificance codes: } 0 * * * 0.001 * * 0.01 * 0.05 .0 .11 \\
\text { Residual standard error: } 0.04208 \text { on } 8 \text { degrees of freedom } \\
\text { Multiple R-squared: } 0.9695 \text {, Adjusted R-squared: } 0.916 \\
\text { F-statistic: } 18.13 \text { on } 14 \text { and } 8 \text { DF, p-value: } 0.0001577\end{array}$}} \\
\hline & & & & & \\
\hline & & & & & \\
\hline & & & & & \\
\hline \multicolumn{3}{|c|}{ Stationary point at response surface } & \multicolumn{2}{|c|}{ Eigen-values } & \\
\hline$a w_{-} w_{i n}$ & 6.987 & & $\lambda_{1}$ & 0.0373 & \\
\hline$a w_{-}$colo & 13.788 & & $\lambda_{2}$ & 0.0064 & \\
\hline$m_{-}$win & 2.495 & & $\lambda_{3}$ & 0.0007 & \\
\hline$c b_{-} w i n$ & 20.615 & & & -0.3249 & \\
\hline
\end{tabular}

The present work shows how to use DOCE to optimize the tuning, by running a dramatically smaller sample (31 experiments). The result of applying DOCE allowed to reach $13.05 \%$ of bad pixels, without the need of Exploratory Analysis. Using DOCE reduces the number of depth maps needed to carry out the study when a large image database is not available. The DOCE methodology itself is independent of the particular algorithms used to generate the disparity maps and it can be used whenever a systematic tunning of process parameters is required.

An improvement from $16.78 \%$ (manual tuning) to $13.05 \%$ in the bad_pixels_all variable might seem negligible at first glance. However, such figures imply a jump of the optimized algorithm of almost 10 positions in the Middlebury Stereo Evaluation ranking. It must be noticed that many algorithms competing in such a rank could benefit from the systematic tunning presented here.

\section{Acknowledgments}

This work has been partially supported by the Spanish Administration Agency CDTI under project CENITVISION 2007-1007, the Colombian Administrative Department of Science, Technology, and Innovation; and the Colombian National Learning Service (COLCIENCIAS - SENA) grant No. 1216-479-22001.

\section{References}

1. Scharstein, D., Szeliski, R.: A taxonomy and evaluation of dense two-frame stereo correspondence algorithms. Int. J. Comput. Vision, 47(1-3):7-42 (2002)

2. Gong, M., Yang, R., Wang, L., Gong, M.. A performance study on different cost aggregation approaches used in realtime stereo matching. Int. J. Comput. Vision, 75:283-296 (2007)

3. Yoon, K., Kweon, I.: Adaptive support-weight approach for correspondence search. IEEE Trans. Pattern Anal. Mach. Intell., 28(4):650 (2006)

4. Congote, J., Barandiaran I., Barandiaran, J., Montserrat, T., Quelen, J., Ferrán, C., Mindan, P., Mur, O., Tarrés, F., Ruiz, O.: Real-time depth map generation architecture for $3 \mathrm{~d}$ videoconferencing. 3DTV-Conference: The True VisionCapture, Transmission and Display of 3D Video (3DTVCON), 2010, 1-4 (2010)

5. Gu, Z., Su, X., Liu, Y., Zhang, Q.: Local stereo matching with adaptive support-weight, rank transform and disparity calibration. Pattern Recogn. Lett., 29:1230-1235 (2008)

6. Hosni, A., Bleyer, M., Gelautz, M., Rhemann, C.: Local stereo matching using geodesic support weights. Proceedings of the 16th IEEE Int. Conf. on Image Processing (ICIP), 2093-2096 (2009)

7. Wang, L., Gong, M., Gong, M., Yang, R.: How far can we go with local optimization in real-time stereo matching. Proceedings of the Third International Symposium on 3D Data Processing, Visualization, and Transmission (3DPVT'06), 129-136 (2006)

8. Fua, P.: A parallel stereo algorithm that produces dense depth maps and preserves image features. Machine Vision and Applications, 6(1):35-49 (1993)

9. Weiss, B.: Fast median and bilateral filtering. ACM Trans. Graph., 25:519-526 (2006)

10. Scharstein, D., Szeliski, R.: High-accuracy stereo depth maps using structured light. IEEE Conference on Computer Vision and Pattern Recognition, 1:195-202 (2003)

11. Scharstein, D., Pal, C.: Learning conditional random fields for stereo. IEEE Conference on Computer Vision and Pattern Recognition, 0:1-8 (2007)

12. Hirschmuller, H., Scharstein, D.: Evaluation of cost functions for stereo matching. IEEE Conference on Computer Vision and Pattern Recognition, 0:1-8 (2007)

13. Tombari, F., Mattoccia, S., Di Stefano, L., Addimanda, E.: Classification and evaluation of cost aggregation methods for stereo correspondence. IEEE Conference on Computer Vision and Pattern Recognition, 1-8 (2008)

14. Daniel, C.: Use of Half-Normal Plots in Interpreting Factorial Two-Level Experiments. Technometrics 1(4):311341(1959)

15. Russell V. Lenth (2009). Response-Surface Methods in R, Using rsm. Journal of Statistical Software, 32(7), 1-17. URL http://www. jstatsoft.org/v32/i07/

16. Scharstein, D., Szeliski, R.: Middlebury stereo vision page. http://vision.middlebury.edu/stereo/ (2007)

17. Douglas C. Montgomery: Design and Analysis of Experiments 7th Edition with Minitab Manual Design \& Analysis of Experiements ISBN 978-0-470-88606-9 April 2010, 2009

18. Peter Dalgaard: Introductory Statistics with R. Ed. 3, Springer Verlag, 2008, ISBN 0387790535.

19. Hoyos, Alejandro; Congote, John; Barandiaran, Inigo; Acosta, Diego; Ruiz, Oscar: Statistical Tuning of AdaptiveWeight Depth Map Algorithm, CAIP 2011. Computer Analysis of Images and Patterns. pp. 563-572. ISBN 978-3642-23677-8. 2011. 